\title{
DUODENO-JEJUNITE PROXIMAL E PLEUROPNEUMONIA EM UM EQUINO - RELATO DE CASO
}

Lucas Lopes Rino Dias"; Renato Bacarin Zavilenski²; Arthur José Schiestl1; Max Gimenez Ribeiro 3

1 - Médico Veterinário, Mestrando do Programa de Pós-graduação em Produção Sustentável e Saúde Animal, Universidade Estadual de Maringá, Umuarama - PR.

E-mail: I.lopesrinodias@gmail.com;

2 - Médico Veterinário, Mestre em Produção Sustentável e Saúde Animal,

Universidade Estadual de Maringá, Umuarama - PR;

3 - Prof.Dr. do Curso de Pós-graduação em Produção Sustentável e Saúde Animal,

Universidade Estadual de Maringá, Umuarama - PR.

Recebido em: 02/10/2017 - Aprovado em: 21/11/2017 - Publicado em: 05/12/2017

DOI: 10.18677/EnciBio_2017B25

\begin{abstract}
O transporte pode predispor a diversas afecções, entre estas a duodeno-jejunite proximal. A duodeno-jejunite proximal é uma afecção grave que pode ocasionar endotoxemia, síndrome da resposta inflamatória sistêmica (SRIS), e sepse. O comprometimento dos mecanismos de defesa pulmonar pode resultar em pleuropneumonia. O objetivo desse trabalho é relatar um caso de Duodeno-jejunite proximal pelo transporte longo de um equino, que resultou em Pleuropneumonia, 0 qual sobreviveu pela correta constatação e intervenção das enfermidades. $O$ animal chegou ao Hospital Veterinário da UEM com histórico de transporte longo e sinais de duodeno-jejunite proximal. Após 14 horas de tratamento intensivo 0 animal apresentou síndrome da resposta inflamatória sistêmica (SRIS), que resultou em sepse e o desenvolvimento de pleuropneumonia bacteriana. Portanto, sendo também medicado para esta enfermidade, e monitorado por exames ultrassonográficos diários, constatando-se o aumento de líquido acompanhado de dispneia, no terceiro dia. Por isso, optou-se pela drenagem do líquido pleural com trocater. Após 20 dias, os valores de fibrinogênio e leucograma estavam normais, assim o animal recebeu alta. Neste caso, a duodeno-jejunite proximal levou a distúrbios sistêmicos que causaram a pleuropneumonia. Estas afecções possuem prognóstico reservado ao ocorrem separadas, e sombrio juntas. Assim, destaca-se o correto monitoramento e intervenções assertivas como fatores determinantes na sobrevivência deste animal.
\end{abstract}

RESUMO

PALAVRAS-CHAVE: Cólica; Efusão pleural; Síndrome da resposta inflamatória sistêmica. 


\begin{abstract}
Very much conditions can be predisposed by transport, including proximal duodenum-jejunitis. Duodenitis-proximal jejunitis is a serious condition that can lead to endotoxemia, systemic inflammatory response syndrome (SIRS), and sepsis. Impaired pulmonary defense mechanisms may result in pleuropneumonia. The objective of this study is to report a case of proximal duodenum-jejunitis due to long transport in an equine, which resulted in Pleuropneumonia, which survived by correct confirmation and intervention of the diseases. The animal arrived at the Veterinary Hospital at the state University of Maringá with a long transport history and signs of proximal duodenum-jejunitis. After 14 hours of intensive treatment the animal presented SIRS, which resulted in sepsis and the development of bacterial pleuropneumonia. Therefore, being also medicated for this disease, and monitored by daily ultrasonographic examinations, which found increased fluid accompanied by dyspnea on the third day. Therefore, pleural fluid drainage was chosen. After 20 days, the values of fibrinogen and leukogram were normal, so the animal was discharged. In this case proximal duodenum-jejunitis led to systemic disorders that caused pleuropneumonia. These conditions have a bad prognosis separately, and somber together. Thus, the correct monitoring and assertive interventions stand out as determining factors in the survival of this animal.
\end{abstract}

KEYWORDS: Colic; Pleural effusion; Systemic inflammatory response syndrome.

\title{
INTRODUÇÃO
}

A maioria dos equinos ao serem transportados são submetidos a condições estressantes que predispõem o estabelecimento de alguns distúrbios, como cólica (NAZARENO et al., 2015) e pleuropneumonia (JOHNS et al., 2017). O transporte de equinos frequentemente causa estresse, bem como ocorre durante o dia e há a privação de água, sendo fatores determinantes para estabelecimento da cólica (DIAS et al., 2013).

A duodeno-jejunite proximal consiste em um processo inflamatório, no qual são observados sinais de dor abdominal, refluxo intestinal, podendo levar à endotoxemia que por sua vez pode causar à Síndrome da Resposta Inflamatória Sistêmica (SRIS) e sepse (TAYLOR, 2015). Quando um patógeno passa pelo trato respiratório superior e acessa o inferior, o desenvolvimento de uma afecção depende dos mecanismos de defesa (GILKERSON et al., 2015), que podem estar comprometidos devido ao acometimento sistêmico por outras afecções, e assim resultar em pleuropneumonia (ARROYO et al., 2017b).

As infecções do trato respiratório com o envolvimento dos brônquios e pulmão denominam-se broncopneumonia, já ao acometer o espaço pleural, pleuropneumonia. Segundo Tomlinson et al. (2015); Johns et al. (2017) as pleuropneumonias com derrames pleurais normalmente são associadas a infecções bacterianas.

A duodeno-jejunite proximal, bem como a pleuropneumonia, são doenças de importância na clínica de equinos pelo prognóstico, que se agrava quando adquire as duas (TAYLOR, 2015; TOMLINSON et al., 2015). O objetivo deste trabalho é relatar um caso de um equino com Duodeno-jejunite devido ao transporte longo, que posterirormente ocasionou Pleuropneumonia, o qual sobreviveu pela correta constatação e intervenção das enfermidades. 


\section{RELATO DE CASO}

Foi atendido no Hospital Veterinário da Universidade Estadual de Maringá (HV-UEM), Campus de Ciências Agrárias de Umuarama, uma égua da raça Mangalarga, de dois anos e meio de idade, de $320 \mathrm{~kg}$ e histórico de longo transporte, $1000 \mathrm{~km}$, por dois dias.

Ela foi transportada do Rio Grande do Sul para a Bahia com outros animais, por meio de um caminhão boiadeiro. No primeiro dia de viagem houve uma parada para pouso no Paraná, onde constatou-se os primeiros sinais clínicos, sendo: apatia, anorexia, respiração abdominal, sentar e levantar, e secreção nasal. Foi medicada para dor, tal medicação desconhecida pelo tutor. No dia seguinte foi mantida em uma propriedade próxima à Umuarama, para ser observada. Após um dia de tratamento os sinais se agravaram, e então foi levada ao HV-UEM na manhã do outro dia.

No HV-UEM demonstrou apatia, inquietação e olhava frequentemente para o flanco. No exame físico as mucosas estavam congestas, em alguns momentos com halo gengival dentário cianótico. Apresentava taquicardia, 64 batimentos/minuto, taquipnéia, 40 movimentos/minuto, e tempo de preenchimento capilar aumentado, 5 segundos, bem como perda da elasticidade no teste de prega cutânea, 4 segundos. $\mathrm{Na}$ auscultação do trato gastrointestinal a motilidade estava ausente, e ao ser aplicada a sondagem nasogástrica houve grande quantidade de refluxo intestinal espontâneo de $\mathrm{pH}$ 7, aproximadamente 15 litros.

Por isso, empregou-se fluidoterapia com Ringer e Lactato $(6 \mathrm{~mL} / \mathrm{kg} / \mathrm{h}$ pela via intravenosa- IV), anti-inflamatório não esteroidal, Flunixina meglumina $(1,1 \mathrm{mg} / \mathrm{kg} \mathrm{IV})$, solução Glicofisiológica $10 \%(1,43 \mathrm{mg} / \mathrm{kg}$, IV) intercalada entre o emprego de Gluconato de cálcio $(0,285 \mathrm{mg} / \mathrm{kg}$, IV), e de Lidocaína $(0,012 \mathrm{mg} / \mathrm{kg}$, IV), ambos diluídos em um litro de solução de $\mathrm{NaCl} 0,9 \%$. A sonda nasogástrica foi mantida enquanto houve refluxo.

Após 14 horas de tratamento o animal apresentou uma repentina piora do quadro, com elevação dos batimentos cardíacos, 66, e frequência respiratória, 50. A mucosa oral tornou-se bem congesta, avermelhada, com halos dentários, o tempo de preenchimento capilar passou a ser maior que 4 segundos, e apresentou febre, $39,4^{\circ} \mathrm{C}$. Devido a isso, empregou-se Dexametasona $(0,15 \mathrm{mg} / \mathrm{kg}$, IV) e Dimetil sulfóxido (DMSO, $0,57 \mathrm{mg} / \mathrm{kg}$, IV), este último diluído em dois litros de solução de $\mathrm{NaCl}$ 0,9\%. Também foram aplicados: Glicose e Gluconato de cálcio.

Passado $1 \mathrm{~h} 20 \mathrm{~m}$ desta intervenção, houve outro quadro de piora, sendo adotada conduta semelhante, com a alteração da dose de Flunixina meglumina para $0,25 \mathrm{mg} / \mathrm{kg}$ quatro vezes ao dia, e a adição de antibióticoterapia duas vezes ao dia: Penicilina (12.000Ul $/ \mathrm{kg}$, intramuscular -IM) e Gentamicina $(6 \mathrm{mg} / \mathrm{kg}, \mathrm{IV})$. O DMSO $(0,5 \mathrm{mg} / \mathrm{kg}$ a $10 \%$, uma vez o dia, IV) e a Dexametasona $(0,05 \mathrm{mg} / \mathrm{kg}$, uma vez ao dia, IV) foram empregados durante uma semana.

Após 21 horas de monitoração e tratamento intensivo, o animal passou a apresentar sibilos e roce pleural severos no pulmão direito, e moderados no esquerdo, descarga nasal sero-sanguinolenta e pleurodinia, suspeitando assim de pleuropneumonia, por agente comensal, com efusão pleural. Por isso, solicitou-se exame ultrassonográfico do tórax e hemograma, e a adição de Metronidazol $(20 \mathrm{mg} / \mathrm{kg}, \mathrm{IV})$ duas vezes ao dia à antibióticoterapia. Como cessou o refluxo intestinal, a sonda foi removida, e o animal foi solto em baia sob observação. A fluidoterapia foi adotada durante o período que o animal esteve internado de forma a impedir a desidratação. 
O hemograma demonstrou aumento do fibrinogênio, 800, aumento significativo de bastonetes $(24 \%, 1.560)$ e baixo número de segmentados $(13 \%$, 245), os demais valores estavam de acordo com a referência para a espécie. Com o exame ultrassonográfico foi possível observar a presença de líquido entre as pleuras visceral e parietal, especialmente no lado direito, bem como alterações de ecogenicidade na superfície pulmonar, sugerindo, efusão pleural e abcessos pulmonares, respectivamente, também observou-se áreas com atelectasia, confirmando a suspeita prévia. A fim de acompanhar a evolução, o exame ultrassonográfico foi instituído diariamente.

No terceiro dia após a chegada do animal, havia dificuldade respiratória com eliminação de catarro purulento. Com exame ultrassonográfico detectou-se aumento significativo da quantidade de líquido efundido, demonstrado pela Figura $1 \mathrm{~A}$, com quantidade superior no lado direito, por isso optou-se pela drenagem, e um outro hemograma. Também foi observada marcante perda de peso, aproximadamente 50kg. Na Figura 1B constata-se o escore de condição corporal ruim deste animal.
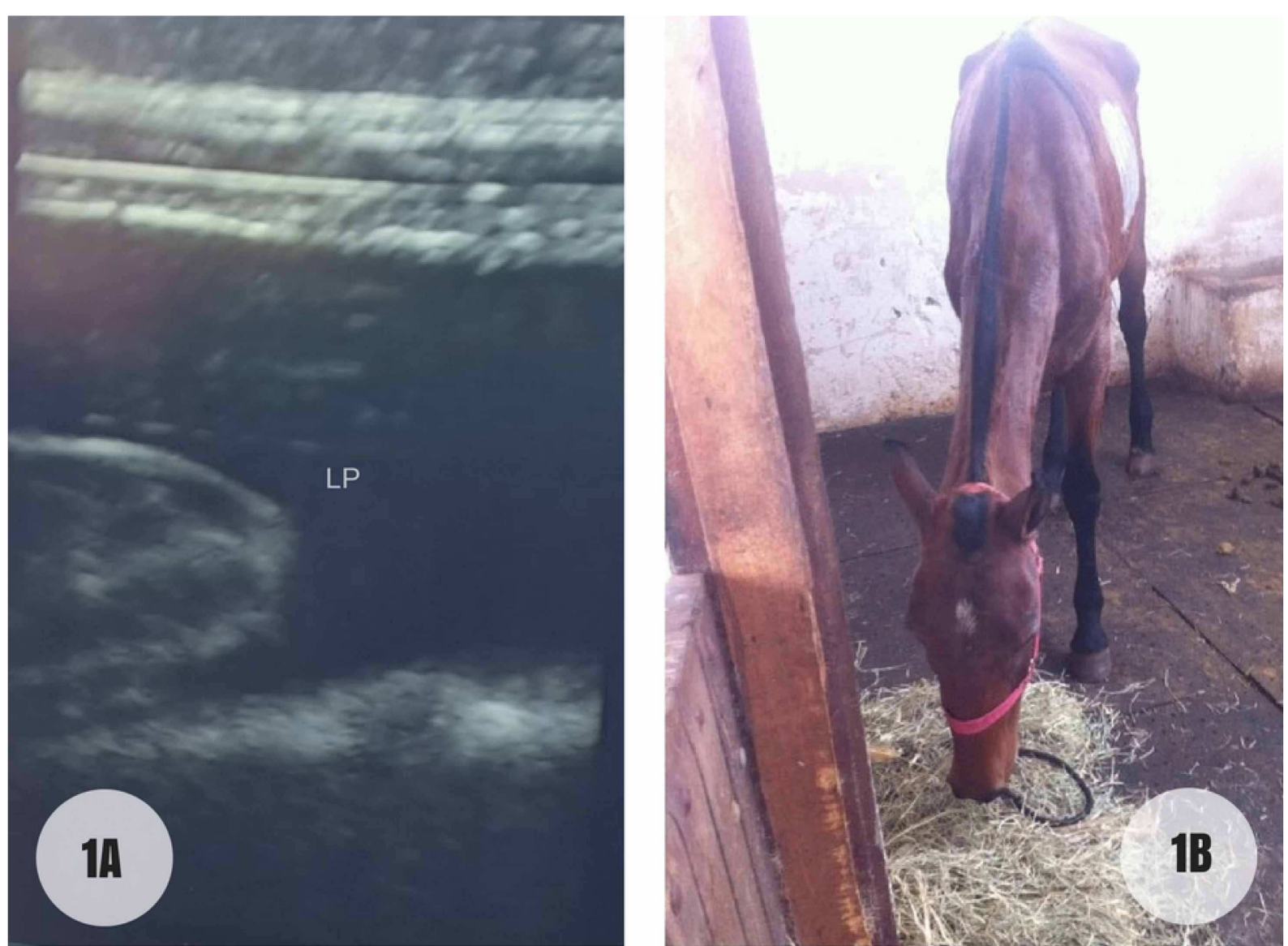

FIGURA 1: No dia três constatou-se aumento do líquido pleural, e marcante perda de peso. A: Líquido Pleural (LP) efundido, nota-se distanciamento entre as pleuras (área anecóica). B: llustração do escore de condição corporal ruim do animal em questão, após o desenvolvimento da pleuropneumonia.

Fonte: Acervo pessoal.

Para a drenagem, realizou-se tricotomia dos pelos adjacentes e antissepsia da pele no nono espaço intercostal. Um tubo plástico de $25 \mathrm{~cm}$ foi acoplado ao 
trocater, ambos estéreis, para evitar influxo de ar ao tórax. A Figura 2A demonstra o momento da drenagem. Para maior efetividade e segurança, o procedimento foi guiado por ultrassom. Com este procedimento, foram drenados 2,5 litros do lado direito e 1,5 litros do lado esquerdo, sendo constatada menor quantidade de fibrina no direito.

Com a análise do líquido, de cada lado, foi possível observar que as características de ambos foram semelhantes. Constatou-se coloração vermelhaalaranjada, como ilustrada pela Figura 2B, turvo, com acentuada quantidade de sedimento, presença de sangue oculto, grande quantidade de hemácias e células nucleadas íntegras em sua maioria, discreta quantidade de células mononucleadas e proteína total alta.
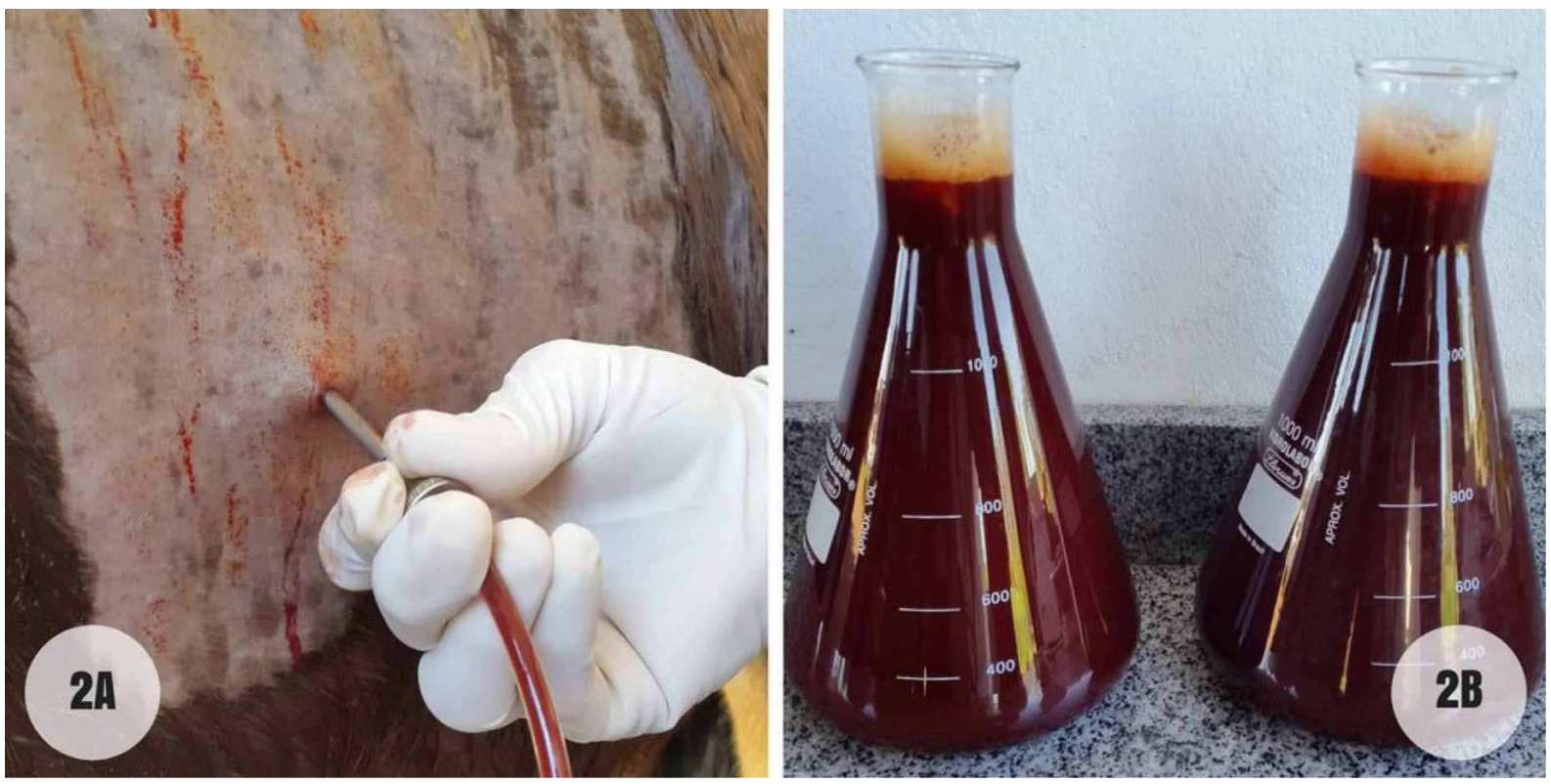

FIGURA 2: Drenagem torácica do líquido pleural. A: Procedimento de trocaterização para a drenagem. B: Erlenmeyer contendo o líquido pleural coletado.

Fonte: Acervo pessoal.

O hemograma passou a apresentar diminuição leve do hematócrito, 29\%, elevado número de leucócitos totais, 23.500, sendo 1.170 bastonetes (5\%) e 15.444 segmentados (66\%). Assim, foi instituído diurético de alça, Furosemida $(1,06 \mathrm{mg} / \mathrm{kg}$, duas vezes ao dia, IV), e espasmolítico e broncodilatador, Clembuterol $(0,1 \mathrm{mg} / \mathrm{kg}$, duas vezes ao dia, pela via oral). Seguiu-se com a antibióticoterapia, cessou a Flunixina meglumina anti-endotoxêmica, e institui-se a dose anti-inflamatória $(1,1 \mathrm{mg} / \mathrm{kg}$, IM, duas vezes ao dia).

No oitavo dia de tratamento as mucosas estavam pálidas. Realizou-se então o hemograma, que demonstrou anemia (18,7\% de hematócrito) normocítica e normocrômica. O fibrinogênio ainda elevado, 800, leucocitose por neutrofilia, 33.800 e 28.730, respectivamente. Por isso, aplicou-se Diproprionato de imidocarb $(2,4 \mathrm{mg} / \mathrm{kg})$ uma vez por dia, durante dois dias), a antibioticoterapia foi mantida, e as aplicações de anti-inflamatório foram cessadas. Com 20 dias de tratamento encerrou-se a antibioticoterapia e o animal recebeu alta médica veterinária.

\section{RESULTADOS E DISCUSSÃO}


O histórico de viagem longa correlacionado aos sinais relatados, sugeriu que o animal teve distúrbio respiratório devido ao estresse e transporte (REUSS; GIGUĖRE, 2015; REUSS; COHEN, 2015), em um primeiro momento, observado pela presença de secreção nasais, associado a um quadro de cólica, constatado pelos sinais de dor abdominal. Esta afecção respiratória ocorreu pelo transporte longo, a cabeça direcionada contra o vento, exposição a diferentes temperaturas e umidades relativa (NAZARENO et al., 2015; HURLEY et al., 2016). Mas logo os sinais passaram, e por isso considerou-se a mesma como branda.

Diferentemente do quadro de cólica, que prosseguiu evoluindo, e por isso o animal foi encaminhado ao HV-UEM. No exame físico foi constatada desidratação moderada, hipotensão, dor abdominal, pelo sequestro de fluidos corporais para o lúmen intestinal, refluxo intestinal, que segundo Taylor (2015) indicam um quadro grave de Duodeno-jejunite proximal. Embora a causa do processo seja desconhecida, suspeita-se que o estresse e a privação de água permitiram a multiplicação de bactérias relacionadas a esta enfermidade no animal, como as do gênero Clostridium spp. (ARROYO et al., 2017a).

A fluidoterapia foi instituída a fim de corrigir a desidratação e melhorar a hipotensão, o anti-inflamatório não esteroidal para modular a resposta inflamatória, controle da dor e ação anti-endotoxêmica, esta última comum em pacientes com quadros clínicos semelhantes ao relatado, sendo procedimentos indicados em situações desse tipo conforme descrito por Taylor (2015).

Níveis baixos de Cálcio frequentemente ocorrem em quadros de cólicas, segundo Dias et al. (2013), que impede o retorno da motilidade e vascularização intestinal normal, por isso o emprego no presente caso. A Lidocaína também possui a função de corrigir a motilidade.

A Glicose foi empregada para fornecer energia para o animal, visto que faziam horas que não se alimentava, e não conseguiria devido ao refluxo. A sondagem nasogástrica conforme Dias et al. (2013) é um procedimento frequente no atendimento de cólica equina, a qual foi realizada e mantida por haver refluxo intestinal constante no estômago. Cabe salientar que a espécie equina não tem o centro do vômito desenvolvido e possui o cárdia bem desenvolvido, impossibilitando assim a eructação e o vômito.

A intervenção inicial proporcionou melhora da condição clínica geral do animal, com o tempo de preenchimento capilar menor que 2", e a frequência respiratória, 12. Embora diminuída, a motilidade melhorou. A coloração da mucosa oral permaneceu sem halos dentários, mas levemente congesta, e os batimentos cardíacos diminuíram para 52. O refluxo intestinal diminuiu em quantidade e frequência.

Os dois episódios de piora clínica mencionados estavam relacionados à SRIS, como resposta à endotoxemia pela cólica, que culminou no restabelecimento de uma infecção pulmonar (TAYLOR, 2015; ILAS, 2017). Segundo Taylor (2015) a supressão da resposta inflamatória, o emprego de fluidoterapia, o combate à endotoxemia e ao agente são os procedimentos adequados para equinos nestas condições. Por isso empregou-se Dexametasona, DMSO, a dose de Flunixina meglumina foi alterada para anti-endotoxêmica e Gentamicina associada a Penicilina.

As medidas empregadas para estabilizar o paciente foram eficazes, embora o mesmo tenha permanecido em condição clínica crítica. O animal permaneceu com 
52 batimentos cardíacos, 36 movimentos respiratórios, congestão de mucosas, halo dentário, e tempo de preenchimento capilar maior que 4.

No presente relato a infecção bacteriana que ocasionou a pleuropneumonia com derrame pleural (TOMLINSON et al., 2015; JOHNS et al., 2017) houve em decorrência do quadro de sepse causado pela duodeno-jejunite proximal, que culminou na infecção de outro órgão (TAYLOR, 2015).

Assim, houve a constatação dos sinais de infecção respiratória que foram, febre, sibilos e roce pleural, letargia, taquipnéia e taquicardia, tempo de preenchimento capilar prolongado, pleurodinia, descarga nasal, dispneia (HILTON; PUSTERIA, 2009; HURLEY et al., 2016; ARROYO et al., 2017b).

A associação do histórico de evolução, sinais clínicos já citados, hemograma (HILTON; PUSTERIA, 2009), aumento do fibrinogênio (JOHNS et al., 2017), imagem ultrassonográfico (HILTON; PUSTERIA, 2009; TOMLINSON et al., 2015; ARROYO et al., 2017b) com alteração na superfície pulmonar condizente com abcesso, espessamento e líquido entre as pleuras, bem como a análise do líquido pleural com contagem celular e proteína total alta (JOHNS et al., 2017), permitiram confirmar o diagnóstico de pleuropneumonia com efusão pleural. Segundo Hurley et al. (2016) a febre alta, ocorrida neste relato, está relacionada à abcessos pulmonares.

Foram drenados 2,5 litros do lado direiro e 1,5 litro do esquerdo de coloração alaranjado, devido à presença de hemácias, turvo pela grande quantidade de sedimentos. A constatação de grande quantidade de hemácias e células nucleadas íntegras, demonstraram que houve lesão endotelial pela resposta inflamatória descontrolada.

Com o exame ultrassonográfico diário observou-se grande aumento de líquido efundido na pleura que afetou a respiração, no terceiro dia de internamento. A instituição da drenagem do fluído pleural permitiu melhora dos movimentos respiratórios, e com a instituição de Furosemida foi possível a eliminação do líquido, sem a necessidade de outra drenagem. Conforme os autores Hilton e Pusteria (2009), Tomlinson et al. (2015) e Arroyo et al. (2017b) essas afecções precisam de mais de uma drenagem, não sendo incomum a necessidade de toracotomia, a manutenção de drenos, ou ainda o uso de agentes fibrinolíticos como estratégias.

Mesmo que os agentes infecciosos do distúrbio respiratório não tenham sido a causa primária, como neste caso, necessitam ser combatidos (WILKINS; LASCOLA, 2015). Devido à impossibilidade de realizar cultura e antibiograma, acrescentou-se Metronidazol à antibióticoterapia pelo seu uso em associação, e a ação contra bactérias gram-negativas anaeróbicas. Segundo Estell et al. (2016) coinfecções com essas bactérias nas pleuropneumonias constitui uma preocupação.

A Flunixina meglumina, o DMSO e a Dexametasona empregados durante uma semana, permitiram o controle da resposta inflamatória, ponto destacado por Taylor (2015) como importante no tratamento de um paciente que teve quadro de SRIS e sepse. O Clembuterol colaborou para melhora na respiração e na eliminação do catarro.

A terapia de suporte é importante no tratamento de pleuropneumonia (TOMLINSON et al., 2015), como a fluidoterapia no presente caso, que permitiu manter o animal hidratado, sendo uma condição essencial para a recuperação de um paciente crítico.

O prognóstico da duodeno-jejunite é reservado à ruim, sendo que se agrava com a ocorrência de SRIS e sepse, tornando-se pior ao ocorrer envolvimento de outro órgão (TAYLOR, 2015) como ocorreu neste caso, que o paciente apresentou 
pleuropneumonia. Segundo Estell et al. (2016) infecção pulmonar por bactérias diminui a perspectiva quando há evidências de SRIS e sepse, e pelas complicações quanto a extensão da lesão, como a pleuropneumonia.

A anemia observada no oitavo dia, 18\% de hematócrito, referiu-se à condição sub-clínica que muitos animais apresentam à parasitas de hemácias, sendo que ao se apresentarem convalescentes ela se torna clínica (PARRA et al., 2014). Por isso empregou-se o quimioterápico Imidocarb. Após três dias as mucosas se apresentavam normocoradas, e houve melhora no hematócrito, 25\%. Também houve leve melhora da condição corporal, batimentos cardíacos, dispneia e frequência respiratória.

Com 10 dias os valores apresentados de fibrinogênio, leucócitos totais e neutrófilos indicavam que a infecção ainda estava presente. $O$ animal apresentavase clinicamente bem, mas só recebeu alta médica veterinária quando o leucograma e fibrinogênio apresentaram-se normais, quando então cessou-se o tratamento. $O$ término do tratamento ocorreu após 20 dias de cuidados médico veterinário.

\section{CONCLUSÃO}

Com este relato observou-se um caso de duodeno-jejunite proximal causada por condições do transporte, que culminou em SRIS e sepse, e resultaram em pleuropneumonia. Essas afecções separadas possuem importância na clínica de equinos pelo prognóstico reservado. Ao ocorrerem por evolução do quadro em um mesmo animal o prognóstico torna-se sombrio. Assim, destacam-se os cuidados intensivos nas primeiras 21 horas, a fim de reverter a duodeno-jejunite proximal e posteriormente SRIS e sepse, bem como o tratamento correto da pleuropneumonia com monitoração constante, como fatores determinantes na sobrevivência do animal.

\section{REFERÊNCIAS}

ARROYO, L. G.; COSTA, M. C.; GUEST, B. B.; PLATTNER, B. L.; LILLIE, B. N. et al. Duodenitis-Proximal Jejunitis in Horses After Experimental Administration of Clostridium difficile Toxins. Journal of veterinary internal medicine, v. 31, n. 1, p. 158-163, 2017a. Disponível em: <http://onlinelibrary.wiley.com/doi/10.1111/jvim.14624/full>. Doi: 10.1111/jvim.14624.

ARROYO, M. G.; SLOVIS, N. M.; MOORE, G. E.; TAYLOR, S. D. Factors Associated with Survival in 97 Horses with Septic Pleuropneumonia. Journal of Veterinary Internal Medicine, v. 31, n. 3, p. 894-900, 2017b. Disponível em: <http://onlinelibrary.wiley.com/doi/10.1111/jvim.14679/full>. Doi: 10.1111/jvim.14679

DIAS, R. V. D. C., BEVILACQUA, P. D., RIBEIRO FILHO, J. D., RIBEIRO JÚNIOR, J. I., \& SOUZA, M. V. D. Avaliação física e laboratorial da síndrome cólica de equinos em parque de vaquejada. Veterinária e Zootecnia, v. 20, n. 4, p. 658-672, 2013.

em: <http://www.fmvz.unesp.br/rvz/index.php/rvz/article/view/581/498>.

ESTELL, K. E.; YOUNG, A.; KOZIKOWSKI, T.; SWAIN, E. A.; BYRNE, B. A. et al. Pneumonia Caused by Klebsiella spp. in 46 Horses. Journal of veterinary internal medicine, v. $30, \quad$ n. 1 , p. 314-321, 2016. Disponível em: <http://onlinelibrary.wiley.com/doi/10.1111/jvim.13653/full>. Doi: 10.1111/jvim.13653. 
GILKERSON, J. R.; BAILEY, K. E.; DIAZ-MÉNDEZ, A.; HARTLEY, C. A. Update on viral diseases of the equine respiratory tract. Veterinary Clinics of North America: Equine Practice, v. 31, n. 1, p. 91-104, 2015. Disónível em: <http://dx.doi.org/10.1016/j.cveq.2014.11.007>. Doi: 10.1016/j.cveq.2014.11.007.

HILTON, H.; PUSTERIA, N. Intrapleural fibrinolytic therapy in the management of septic pleuropneumonia in a horse. Veterinary Record, v. 164, n. 18, p. 558-559, $2009 . \quad$ Disponível em: <http://veterinaryrecord.bmj.com/content/vetrec/164/18/558.full.pdf>. Doi: 10.1136/vr.164.18.558.

HURLEY, M. J.; RIGGS, C. M.; COGGER, N.; ROSANOWSKI, S. M. The incidence and risk factors for shipping fever in horses transported by air to Hong Kong: Results from a 2-year prospective study. The Veterinary Journal, v. 214, p. 34-39, 2016. Disponível em: <http://dx.doi.org/10.1016/j.tvjl.2016.01.006>. Doi: 10.1016/j.tvjl.2016.01.006.

ILAS - INSTITUTO LATINO AMERICANO DE SEPSE. Atendimento ao paciente adulto com sepse / choque séptico. Implementação de protocolo gerenciado de sepse protocolo clínico. jun. 2017. Disponível em: <http://www.ilas.org.br/assets/arquivos/ferramentas/protocolo-de-tratamento.pdf>.

JOHNS, I.; MARR, C.; DURHAM, A.; MAIR, T.; MCPARLAND, T. Causes of pleural effusions in horses resident in the UK. Equine Veterinary Education, v. 29, n. 3, p. 144-148, 2017. Disponível em: <http://onlinelibrary.wiley.com/doi/10.1111/eve.12569/full>. Doi: 10.1111/eve.12569

NAZARENO, A. C.; DA SILVA, I. J. O.; FERNANDES, D. P. B. Ambiência no transporte de equinos e os efeitos nas respostas ao estresse. Journal of Animal Behaviour and Biometeorology, v. 3, n. 3, p. 73-80, 2015. Disponível em: <http://dx.doi.org/10.14269/2318-1265/jabb.v3n3p73-80>. Doi: 10.14269/23181265/jabb.v3n3p73-80.

PARRA, A. C.; PIOTTO, M. A.; FRESCHI, C. R.; MACHADO, R. Z.; FERNANDES, W. R. Avaliação comparativa de métodos de ensaio imunoenzimático para theileria equi de amostras de soro equino do estado de São Paulo. Ars Veterinaria, v. 30, n. 1, p. 42-47, 2014. Disponível em: <http://dx.doi.org/10.15361/21750106.2014v30n1p42-47>. Doi: 10.15361/2175-0106.2014v30n1p42-47.

REUSS, S. M.; COHEN, N. D. Update on bacterial pneumonia in the foal and weanling. Veterinary Clinics: Equine Practice, v. 31, n. 1, p. 121-135, 2015. Disponível em: <http://dx.doi.org/10.1016/j.cveq.2014.11.004>. Doi: 10.1016/j.cveq.2014.11.004.

REUSS, S. M.; GIGUÈRE, S. Update on bacterial pneumonia and pleuropneumonia in the adult horse. Veterinary Clinics: Equine Practice, v. 31, n. 1, p. 105-120, 2015. Disponível em: <http://dx.doi.org/10.1016/j.cveq.2014.11.002>. Doi: 10.1016/j.cveq.2014.11.002. 
TAYLOR, S. A review of equine sepsis. Equine Veterinary Education, v. 27, n. 2, p. 2015.

Disponível

em: <http://onlinelibrary.wiley.com/doi/10.1111/eve.12290/epdf>.

Doi: 10.1111/eve.12290.

TOMLINSON, J. E.; REEF, V. B.; BOSTON, R. C.; JOHNSON, A. L. The association of fibrinous pleural effusion with survival and complications in horses with pleuropneumonia (2002-2012): 74 Cases. Journal of veterinary internal medicine, v. $29, \quad$ n. $5, \quad$ p. 1410-1417, 2015. Disponível em: <http://onlinelibrary.wiley.com/doi/10.1111/jvim.13591/full>. Doi: 10.1111/jvim.13591.

WILKINS, P. A.; LASCOLA, K. M. Update on interstitial pneumonia. Veterinary Clinics of North America: Equine Practice, v. 31, n. 1, p. 137-157, 2015. Disponível em: <http://dx.doi.org/10.1016/j.cveq.2014.11.006>. Doi: 10.1016/j.cveq.2014.11.006. 